\title{
Some sharp integral inequalities involving partial derivatives
}

\author{
Chang-Jian Zhao ${ }^{1 *}$, Wing-Sum Cheung ${ }^{2}$ and Mihály Bencze ${ }^{3}$
}

\author{
* Correspondence: chjzhao@163. \\ com \\ ${ }^{1}$ Department of Mathematics, \\ China Jiliang University, Hangzhou \\ 310018, P. R. China \\ Full list of author information is \\ available at the end of the article
}

\author{
Abstract \\ The main purpose of the present article is to establish some new sharp integral \\ inequalities in $2 n$ independent variables. Our results in special cases yield some of \\ the recent results on Pachpatter, Agarwal and Sheng's inequalities and provide some \\ new estimates on such types of inequalities. \\ Mathematics Subject Classification 2000: 26D15.
}

Keywords: Cauchy-Schwarz's inequality, Pachpatte's inequality, Hölder integral inequality, the arithmetic-geometric means inequality

\section{Introduction}

Inequalities involving functions of $n$ independent variables, their partial derivatives, integrals play a fundamental role in establishing the existence and uniqueness of initial and boundary value problems for ordinary and partial differential equations as well as difference equations [1-10]. Especially, in view of wider applications, inequalities due to Agarwal, Opial, Pachpatte, Wirtinger, Poincaréand et al. have been generalized and sharpened from the very day of their discover. As a matter of fact these now have become research topic in their own right [11-14]. In the present article we shall use the same method of Agarwal and Sheng [15], establish some new estimates on these types of inequalities involving $2 n$ independent variables. We further generalize these inequalities which lead to result sharp than those currently available. An important characteristic of our results is that the constants in the inequalities are explicit.

\section{Main results}

Let $R$ be the set of real numbers and $\mathbb{R}^{n}$ the $n$-dimensional Euclidean space. Let $E, E^{\prime}$ be a bounded domain in $R^{n}$ defined by $E \times E^{\prime}=\prod_{i=1}^{n}\left[a_{i}, b_{i}\right] \times\left[c_{i}, d_{i}\right], i=1, \ldots, n$. For $x_{i}, y_{i} \in R, i=1, \ldots, n,(x, y)=\left(x_{1}, \ldots, x_{n}, y_{1}, \ldots, y_{n}\right)$ is a variable point in $E \times E^{\prime}$ and $d x d y$ $=d x_{1} \ldots d x_{n} d y_{1} \ldots d y_{n}$. For any continuous real-valued function $u(x, y)$ defined on $E \times$ $E^{\prime}$ we denote by $\int_{E} \int_{E^{\prime}} u(x, y) d x d y$ the $2 n$-fold integral

$$
\int_{a_{1}}^{b_{1}} \ldots \int a_{n}{ }^{b_{n}} \int c_{1}{ }^{d_{1}} \ldots \int c_{n}{ }^{d_{n}} u\left(x_{1}, \ldots, x_{n}, y_{1}, \ldots, y_{n}\right) d x_{1} \ldots d x_{n} d y_{1} \ldots d y_{n}
$$


and for any $(x, y) \in E \times E^{\prime}, \int E(x) \int E^{\prime}(x) u(s, t) d s d t$ is the $2 n$-fold integral

$$
\int a_{1}{ }^{x_{1}} \ldots \int a_{n}^{x_{n}} \int c_{1}^{\gamma_{1}} \ldots \int c_{n}^{\gamma_{n}} u\left(s_{1}, \ldots, s_{n}, t_{1}, \ldots, t_{n}\right) d x_{1} \ldots d s_{n} d t_{1} \ldots d t_{n}
$$

We represent by $F\left(E \times E^{\prime}\right)$ the class of continuous functions $u(x, y): E \times E^{\prime} \rightarrow \mathbb{R}$, for each $i, 1 \leq i \leq n$,

$$
\left.u(x, y)\right|_{x_{i}=a_{i}}=0,\left.u(x, y)\right|_{y_{i}=c_{i}}=0,\left.u(x, y)\right|_{x_{i}=b_{i}}=0,\left.u(x, y)\right|_{y_{i}=d_{i}}=0,(i=1, \ldots, n)
$$

the class $F\left(E \times E^{\prime}\right)$ is denoted as $G\left(E \times E^{\prime}\right)$.

Theorem 2.1. Let $l, \mu, \lambda \geq 1$, be given real numbers such that $\frac{1}{\mu}+\frac{1}{\lambda}=1$. Further, let $u(x, y) \in G\left(E \times E^{\prime}\right)$. Then, the following inequality holds

$$
\begin{aligned}
\int E \int E^{\prime}|u(x, y)|^{l} d x d y \leq & \frac{1}{2 n}\left(\sum_{i=1}^{n}\left[\left(b_{i}-a_{i}\right)\left(c_{i}-d_{i}\right)\right]^{\mu}\right)^{1 / \mu}\left(\int E \int E^{\prime}|u(x, y)|^{(l-1) \mu} d x d y\right)^{1 / \mu} \\
& \times\left(\int E \int E^{\prime}\|\operatorname{grad} u(x, y)\|_{\lambda}^{\lambda} d x d y\right)^{1 / \lambda}
\end{aligned}
$$

where

$$
\|\operatorname{grad} u(x, y)\|_{\lambda}=\left(\sum_{i=1}^{n}\left|\frac{\partial^{2}}{\partial x_{i} \partial y_{i}} u(x, y)\right|^{\lambda}\right)^{1 / \lambda} .
$$

Proof. For each fixed $i, 1 \leq i \leq n$, in view of

$$
\left.u(x, y)\right|_{x_{i}=a_{i}}=0,\left.u(x, y)\right|_{y_{i}=c_{i}}=0,\left.u(x, y)\right|_{x_{i}=b_{i}}=0,\left.u(x, y)\right|_{y_{i}=d_{i}}=0,(i=1, \ldots, n)
$$

we have

$$
u^{l}(x, y)=u^{l-1}(x, y) \int a_{i}^{x_{i}} \int c_{i}^{y_{i}} \frac{\partial^{2}}{\partial s_{i} \partial t_{i}} u\left(x, y ; s_{i}, t_{i}\right) d s_{i} d t_{i}
$$

and

$$
u^{l}(x, y)=u^{l-1}(x, y) \int x_{i}^{b_{i}} \int y_{i} d_{i} \frac{\partial^{2}}{\partial s_{i} \partial t_{i}} u\left(x, y ; s_{i}, t_{i}\right) d s_{i} d t_{i}
$$

where

$$
u\left(x, y ; s_{i}, t_{i}\right)=u\left(x_{1}, \ldots, x_{i-1}, s_{i}, x_{i+1}, \ldots, x_{n}, y_{1}, \ldots, y_{i-1}, t_{i}, y_{i+1}, \ldots, y_{n}\right) .
$$

Hence, from (2.2) and (2.3) and in view of the arithmetic-geometric means inequality and Hölder inequality with indices $\mu$ and $\lambda$, it follows that

$$
\begin{gathered}
|u(x, y)|^{l} \leq \frac{1}{2}|u(x, y)|^{l-1} \int a_{i}^{b_{i}} \int c_{i} d_{i}\left|\frac{\partial^{2}}{\partial s_{i} \partial t_{i}} u\left(x, y ; s_{i}, t_{i}\right)\right| d s_{i} d t_{i} \\
\leq \frac{1}{2}|u(x, y)|^{l-1}\left[\left(b_{i}-a_{i}\right)\left(c_{i}-d_{i}\right)\right]^{1 / \mu}\left(\int a_{i}{ }^{x_{i}} \int c_{i}^{y_{i}}\left|\frac{\partial^{2}}{\partial s_{i} \partial t_{i}} u\left(x, y ; s_{i}, t_{i}\right)\right|^{\lambda} d s_{i} d t_{i}\right)^{1 / \lambda} .
\end{gathered}
$$

Now, summing the inequalities (2.4) for $1 \leq i \leq n$, integrating over $E \times E^{\prime}$ and applying Holder inequality with indices $\mu$ and $\lambda$ two times, we get 


$$
\begin{gathered}
\int E \int E^{\prime}|u(x, y)|^{l} d x d y \leq \frac{1}{2 n} \sum_{i=1}^{n}\left[\left(b_{i}-a_{i}\right)\left(c_{i}-d_{i}\right)\right]^{1 / \mu} \\
\times \int E \int E^{\prime}|u(x, y)|^{l-1}\left(\int a_{i}^{b_{i}} \int c_{i} d_{i}\left|\frac{\partial^{2}}{\partial s_{i} \partial t_{i}} u\left(x, y ; s_{i}, t_{i}\right)\right|^{\lambda} d s_{i} d t_{i}\right)^{1 / \lambda} d x d y \\
\leq \frac{1}{2 n}\left(\int E \int E^{\prime}|u(x, y)|^{(l-1) \mu} d x d y\right)^{1 / \mu} \sum_{i=1}^{n}\left[\left(b_{i}-a_{i}\right)\left(c_{i}-d_{i}\right)\right]^{1 / \mu} \\
\times\left(\int E \int E^{\prime} \int a_{i}{ }^{b_{i}} \int c_{i}{ }^{d_{i}}\left|\frac{\partial^{2}}{\partial s_{i} \partial t_{i}} u\left(x, y ; s_{i}, t_{i}\right)\right|^{\lambda} d s_{i} d t_{i} d x d y\right)^{1 / \lambda} \\
\leq \frac{1}{2 n}\left(\int E \int E^{\prime}|u(x, y)|^{(l-1) \mu} d x d y\right)^{1 / \mu} \sum_{i=1}^{n}\left[\left(b_{i}-a_{i}\right)\left(c_{i}-d_{i}\right)\right]^{1 / \mu+1 / \lambda} \\
\times\left(\int E \int E^{\prime}\left|\frac{\partial^{2}}{\partial x_{i} \partial y_{i}} u(x, y)\right|^{\lambda} d x d y\right)^{1 / \lambda} \\
\leq \frac{1}{2 n}\left(\int E \int E^{\prime}|u(x, y)|^{(l-1) \mu} d x d y\right)^{1 / \mu}\left(\sum_{i=1}^{n}\left[\left(b_{i}-a_{i}\right)\left(c_{i}-d_{i}\right)\right]^{\mu}\right)^{1 / \mu} \\
\times\left(\int E \int E^{\prime}\|\operatorname{grad} u(x, y)\|_{\lambda}^{\lambda} d x d y\right)^{1 / \lambda},
\end{gathered}
$$

where

$$
\|\operatorname{grad} u(x, y)\|_{\lambda}=\left(\sum_{i=1}^{n}\left|\frac{\partial^{2}}{\partial x_{i} \partial y_{i}} u(x, y)\right|^{\lambda}\right)^{1 / \lambda} .
$$

The proof is complete.

Remark 2.1. Let $u(x, y)$ reduce to $u(x)$ in (2.1) and with suitable modifications, then (2.1) becomes

$$
\begin{aligned}
\int E|u(x)|^{(l) \mu} d x \leq & \frac{1}{2 n}\left(\int E|u(x)|^{(l-1) \mu} d x\right)^{1 / \mu}\left(\sum_{i=1}^{n}\left(b_{i}-a_{i}\right)^{\mu}\right)^{1 / \mu} \\
& \times\left(\int E\|\operatorname{grad} u(x)\|_{\lambda}^{\lambda} d x\right)^{1 / \lambda},
\end{aligned}
$$

where

$$
\|\operatorname{grad} u(x)\|_{\lambda}=\left(\sum_{i=1}^{n}\left|\frac{\partial}{\partial x_{i}} u(x)\right|^{\lambda}\right)^{1 / \lambda} .
$$

This is just a important inequality which was given by Agarwal and Sheng [15].

Remark 2.2. For the given real numbers $l_{k} \geq 0,1 \leq k \leq r$, such that $r l_{k} \geq 1$, the arithmetic-geometric means inequality and (2.1) gives

$$
\begin{gathered}
\int E \int E^{\prime} \prod_{k=1}^{r}\left|u_{k}(x, y)\right|^{l_{k}} d x d y \leq \frac{1}{r} \sum_{k=1}^{r} \int E \int E^{\prime}\left|u_{k}(x, y)\right|^{r l_{k}} d x d y \\
\leq \frac{1}{2 n r}\left(\sum_{i=1}^{n}\left[\left(b_{i}-a_{i}\right)\left(c_{i}-d_{i}\right)\right]^{\mu}\right)^{1 / \mu} \sum_{k=1}^{r}\left(\int E \int E^{\prime}\left|u_{k}(x, y)\right|^{\left(r l_{k}-1\right) \mu} d x d y\right)^{1 / \mu} \\
\times\left(\int E \int E^{\prime}\left\|\operatorname{grad} u_{k}(x, y)\right\|_{\lambda}^{\lambda} d x d y\right)^{1 / \lambda} .
\end{gathered}
$$


This is just a general form of the following result which was given by Agarwal and Sheng [15].

$$
\begin{aligned}
\int E \prod_{k=1}^{r}\left|u_{k}(x)\right|^{l_{k}} d x & \leq \frac{1}{2 n r}\left(\sum_{i=1}^{n}\left(b_{i}-a_{i}\right)^{\mu}\right)^{1 / \mu} \sum_{k=1}^{r}\left(\int E\left|u_{k}(x)\right|^{\left(r l_{k}-1\right) \mu} d x\right)^{1 / \mu} \\
& \times\left(\int E\left\|\operatorname{grad} u_{k}(x)\right\|_{\lambda}^{\lambda} d x\right)^{1 / \lambda},
\end{aligned}
$$

where

$$
\left\|\operatorname{grad} u_{k}(x)\right\|_{\lambda}=\left(\sum_{i=1}^{n}\left|\frac{\partial}{\partial x_{i}} u(x)\right|^{\lambda}\right)^{1 / \lambda} .
$$

Remark 2.3. In particular, for $l_{k}=\left(p_{k}+2\right) /(2 r), p_{k} \geq 1,1 \leq k \leq r, \mu=\lambda=2$, the inequality (2.5) reduces to

$$
\begin{gathered}
\int E \int E^{\prime}\left(\prod_{k=1}^{r}\left|u_{k}(x, y)\right|^{\left(p_{k}+2\right) / 2}\right)^{1 / r} d x d y \\
\leq \frac{1}{2 n r}\left(\sum_{i=1}^{n}\left[\left(b_{i}-a_{i}\right)\left(c_{i}-d_{i}\right)\right]^{2}\right)^{1 / 2} \sum_{k=1}^{r}\left(\int E \int E^{\prime}|u(x, y)|^{p_{k}} d x d y\right)^{1 / 2} \\
\times\left(\int E \int E^{\prime}\left\|\operatorname{grad} u_{k}(x, y)\right\|_{2}^{2} d x d y\right)^{1 / 2} .
\end{gathered}
$$

This is just a general form of the following result which was given by Agarwal and Sheng [15].

$$
\begin{gathered}
\int E\left(\prod_{k=1}^{r}\left|u_{k}(x)\right|^{\left(p_{k}+2\right) / 2}\right)^{1 / r} d x \\
\leq \frac{1}{2 n r}\left(\sum_{i=1}^{n}\left(b_{i}-a_{i}\right)^{2}\right)^{1 / 2} \sum_{k=1}^{r}\left(\int E|u(x)|^{p_{k}} d x\right)^{1 / 2}\left(\int E\left\|\operatorname{grad} u_{k}(x)\right\|_{2}^{2} d x\right)^{1 / 2} .
\end{gathered}
$$

On the other hand, the above inequality with the right-hand side multiplied by $\left(\prod_{k=1}^{r}\left(\left(p_{k}+2\right) / 2\right)\right)^{1 / r}$ and the term $\left(\sum_{i=1}^{n}\left(b_{i}-a_{i}\right)^{2}\right)^{1 / 2}$ replace by $\sqrt{n} \beta$ has been proved by Pachpatte [16].

Remark 2.4. If $u(x, y)$ reduce to $u(x)$ in (2.1), then the inequality (2.1) and its particular case $l \geq 2, \mu=\lambda=2$ with the right-hand side multiplied by $l$ have been separately proved by Pachpatte in [17].

Theorem 2.2. Let $\lambda \geq 1$ and $u(x, y) \in G\left(E \times E^{\prime}\right)$. Then, the following inequality holds

$$
\begin{gathered}
\int E \int E^{\prime}|u(x, y)|^{2 \lambda} d x d y \leq \frac{\pi \lambda^{2} \beta^{2} \alpha^{2}}{128 n}\left(\int E \int E^{\prime}|u(x, y)|^{2 \lambda} d x d y\right)^{(\lambda-1) / \lambda} \\
\times\left(\int E \int E^{\prime} \sum_{i=1}^{n}\left|\frac{\partial^{2} u}{\partial s_{i} \partial t_{i}}+(\lambda-1) \frac{1}{u(x, y)} \frac{\partial u}{\partial x_{i}} \frac{\partial u}{\partial y_{i}}\right|^{2 \lambda} d x d y\right)^{1 / \lambda}
\end{gathered}
$$

where $\beta=\max _{1 \leq i \leq n}\left(b_{i}-a_{i}\right)$ and $\alpha=\max _{1 \leq i \leq n}\left(d_{i}-c_{i}\right)$. 
Proof. For each fixed $i, 1 \leq i \leq n$, we obtain that

$$
u^{\lambda}(x, y)=\lambda \int a_{i}^{x_{i}} \int c_{i}^{y_{i}}\left[u^{\lambda-1}\left(x, y ; s_{i}, t_{i}\right) \frac{\partial^{2} u}{\partial s_{i} \partial t_{i}}+(\lambda-1) u^{\lambda-2}\left(x, y ; s_{i}, t_{i}\right) \frac{\partial u}{\partial s_{i}} \frac{\partial u}{\partial t_{i}}\right] d s_{i} d t_{i}
$$

and hence from the Cauchy-Schwarz inequality, it follows that

$$
\begin{gathered}
|u(x, y)|^{\lambda} \leq \lambda^{2}\left(x_{i}-a_{i}\right)\left(y_{i}-c_{i}\right) \\
\times \int a_{i}^{x_{i}} \int c_{i}^{y_{i}}\left|y^{\lambda-1}\left(x, y ; s_{i}, t_{i}\right) \frac{\partial^{2} u}{\partial s_{i} \partial t_{i}}+(\lambda-1) u^{\lambda-2}\left(x, y ; s_{i}, t_{i}\right) \frac{\partial u}{\partial s_{i}} \frac{\partial u}{\partial t_{i}}\right|^{2} d s_{i} d t_{i}
\end{gathered}
$$

and similarly,

$$
\begin{gathered}
|u(x, y)|^{\lambda} \leq \lambda^{2}\left(b_{i}-x_{i}\right)\left(d_{i}-y_{i}\right) \\
\times \int x_{i} b_{i} \int y_{i} d_{i}\left|u^{\lambda-1}\left(x, y ; s_{i}, t_{i}\right) \frac{\partial^{2} u}{\partial s_{i} \partial t_{i}}+(\lambda-1) u^{\lambda-2}\left(x, y ; s_{i}, t_{i}\right) \frac{\partial u}{\partial s_{i}} \frac{\partial u}{\partial t_{i}}\right|^{2} d s_{i} d t_{i}
\end{gathered}
$$

Hence, multiplying (2.7) and (2.8) and in view of using the arithmetic-geometric means inequality, summing the resulting inequalities for $1 \leq i \leq n$, and then integrating over $E \times E^{\prime}$, to obtain

$$
\begin{gathered}
\int E \int E^{\prime}|u(x, y)|^{2 \lambda} d x d y \leq \frac{\lambda^{2}}{2 n} \int E \int E^{\prime}\left\{\sum_{i=1}^{n}\left[\left(x_{i}-a_{i}\right)\left(y_{i}-c_{i}\right)\left(b_{i}-x_{i}\right)\left(d_{i}-y_{i}\right)\right]^{1 / 2}\right. \\
\left.\times \int a_{i}^{b_{i}} \int c_{i} d_{i}\left|u^{\lambda-1}\left(x, y ; s_{i}, t_{i}\right) \frac{\partial^{2} u}{\partial s_{i} \partial t_{i}}+(\lambda-1) u^{\lambda-2}\left(x, y ; s_{i}, t_{i}\right) \frac{\partial u}{\partial s_{i}} \frac{\partial u}{\partial t_{i}}\right|^{2} d s_{i} d t_{i}\right\} d x d y \\
=\frac{\lambda^{2}}{2 n} \sum_{i=1}^{n} \int a_{i}{ }^{b_{i}} \int c_{i} d_{i}\left[\left(x_{i}-a_{i}\right)\left(y_{i}-c_{i}\right)\left(b_{i}-x_{i}\right)\left(d_{i}-y_{i}\right)\right]^{1 / 2} d x_{i} d y_{i} \\
\times \int E \int E^{\prime}\left|y^{\lambda-1}(x, y) \frac{\partial^{2} u}{\partial s_{i} \partial t_{i}}+(\lambda-1) u^{\lambda-2}(x, y) \frac{\partial u}{\partial x_{i}} \frac{\partial u}{\partial y_{i}}\right|^{2} d x d y \\
\leq \frac{\pi \lambda^{2} \beta^{2} \alpha^{2}}{128 n} \int E \int E^{\prime} \sum_{i=1}^{n}\left|u^{\lambda-1}(x, y) \frac{\partial^{2} u}{\partial s_{i} \partial t_{i}}+(\lambda-1) u^{\lambda-2}(x, y) \frac{\partial u}{\partial x_{i}} \frac{\partial u}{\partial y_{i}}\right|^{2} d x d y,
\end{gathered}
$$

where $\beta=\max _{1 \leq i \leq n}\left(b_{i}-a_{i}\right)$ and $\alpha=\max _{1 \leq i \leq n}\left(d_{i}-c_{i}\right)$.

Hence, using Hölder inequality with indices $\lambda$ and $\lambda /(\lambda-1)$ in right-hand side of above inequality, we have

$$
\begin{gathered}
\int E \int E^{\prime}|u(x, y)|^{2 \lambda} d x d y \leq \frac{\pi \lambda^{2} \beta^{2} \alpha^{2}}{128 n}\left(\int E \int E^{\prime}|u(x, y)|^{2 \lambda} d x d y\right)^{(\lambda-1) / \lambda} \\
\quad \times\left(\int E \int E^{\prime} \sum_{i=1}^{n}\left|\frac{\partial^{2} u}{\partial s_{i} \partial t_{i}}+(\lambda-1) \frac{1}{u(x, y)} \frac{\partial u}{\partial x_{i}} \frac{\partial u}{\partial y_{i}}\right|^{2 \lambda} d x d y\right)^{1 / \lambda}
\end{gathered}
$$

The proof is complete.

Remark 2.5. Let $u(x, y)$ reduce to $u(x)$ in (2.6) and with suitable modifications, then (2.6) becomes the following Agarwal and Sheng [15] inequality.

$$
\int E|u(x)|^{2 \lambda} d x \leq \frac{\pi \lambda^{2} \beta^{2}}{16 n}\left(\int E|u(x)|^{2 \lambda} d x\right)^{(\lambda-1) / \lambda}\left(\int E\|\operatorname{grad} u(x)\|_{2}^{2 \lambda} d x\right)^{1 / \lambda}
$$

where $\beta=\max _{1 \leq i \leq n}\left(b_{i}-a_{i}\right)$. 
Theorem 2.3. Let $l \geq 0, m \geq 1$ be given real numbers, and let $u(x, y) \in G\left(E \times E^{\prime}\right)$.

Then, the following inequality holds

$$
\begin{gathered}
\int E \int E^{\prime}|u(x, y)|^{l+m} d x d y \leq \frac{1}{n}\left(\frac{m+l}{2 m}\right)^{m} \sum_{i=1}^{n}\left[\left(b_{i}-a_{i}\right)\left(d_{i}-c_{i}\right)\right]^{m} \\
\times \int E \int E^{\prime}\left|u^{l / m}(x, y) \frac{\partial^{2} u}{\partial x_{i} \partial y_{i}}+\frac{l}{m} u^{(l / m-1)}(x, y) \frac{\partial u}{\partial x_{i}} \frac{\partial u}{\partial y_{i}}\right|^{m} d x d y .
\end{gathered}
$$

Proof. For each fixed $i, 1 \leq i \leq n$, we obtain that

$$
\begin{gathered}
u^{l+m}(x, y)=\frac{m+l}{m}[u(x, y)]^{(m-1)(l+m) / m} \\
\times \int a_{i}^{x_{i}} \int c_{i} y_{i}\left[u^{l / m}\left(x, y ; s_{i}, t_{i}\right) \frac{\partial^{2} u}{\partial s_{i} \partial t_{i}}+\frac{l}{m} u^{(l / m-1)}\left(x, y ; s_{i}, t_{i}\right) \frac{\partial u}{\partial s_{i}} \frac{\partial u}{\partial t_{i}}\right] d s_{i} d t_{i}
\end{gathered}
$$

and, hence, it follows that

$$
\begin{gathered}
|u(x, y)|^{l+m} \leq \frac{m+l}{m}|u(x, y)|^{(m-1)(l+m) / m} \\
\times \int a_{i}^{x_{i}} \int c_{i}^{y_{i}}\left|u^{l / m}\left(x, y ; s_{i}, t_{i}\right) \frac{\partial^{2} u}{\partial s_{i} \partial t_{i}}+\frac{l}{m} u^{(l / m-1)}\left(x, y ; s_{i}, t_{i}\right) \frac{\partial u}{\partial s_{i}} \frac{\partial u}{\partial t_{i}}\right| d s_{i} d t_{i}
\end{gathered}
$$

and, similarly,

$$
\begin{gathered}
|u(x, y)|^{l+m} \leq \frac{m+l}{m}|u(x, y)|^{(m-1)(l+m) / m} \\
\times \int x_{i} b_{i} \int y_{i} d_{i}\left|u^{l / m}\left(x, y ; s_{i}, t_{i}\right) \frac{\partial^{2} u}{\partial s_{i} \partial t_{i}}+\frac{l}{m} u^{(l / m-1)}\left(x, y ; s_{i}, t_{i}\right) \frac{\partial u}{\partial s_{i}} \frac{\partial u}{\partial t_{i}}\right| d s_{i} d t_{i} .
\end{gathered}
$$

Now, adding (2.9) and (2.10) and integrating the resulting inequality from $a_{i}$ to $b_{i}$ and $c_{i}$ to $d_{i}$, respectively. Then

$$
\begin{gathered}
\int a_{i}{ }^{b_{i}} \int c_{i}{ }^{d_{i}}|u(x, y)|^{l+m} d x_{i} d y_{i} \leq \frac{m+l}{2 m}\left(\int a_{i}^{b_{i}} \int c_{i}^{d_{i}}|u(x, y)|^{(m-1)(l+m) / m} d x_{i} d y_{i}\right) \\
\times \int a_{i}{ }^{b_{i}} \int c_{i}{ }^{d_{i}}\left|u^{l / m}(x, y) \frac{\partial^{2} u}{\partial x_{i} \partial y_{i}}+\frac{l}{m} u^{(l / m-1)}(x, y) \frac{\partial u}{\partial x_{i}} \frac{\partial u}{\partial y_{i}}\right| d x_{i} d y_{i} .
\end{gathered}
$$

Next in each integral of the right-hand side of the above inequality we apply Hölder inequality with indices $m$ and $m /(m-1)$, to get

$$
\begin{gathered}
\int a_{i}^{b_{i}} \int c_{i}{ }^{d_{i}}|u(x, y)|^{l+m} d x_{i} d y_{i} \leq \frac{m+l}{2 m}\left(\int a_{i}{ }^{b_{i}} \int c_{i}{ }^{d_{i}}|u(x, y)|^{l+m} d x_{i} d y_{i}\right)^{(m-1) / m} \\
\times\left[\left(b_{i}-a_{i}\right)\left(d_{i}-c_{i}\right)\right]^{1 / m}\left[\left(b_{i}-a_{i}\right)\left(d_{i}-c_{i}\right)\right]^{(m-1) / m} \\
\times\left(\int a_{i}{ }^{b_{i}} \int c_{i}{ }^{d_{i}}\left|u^{l / m}(x, y) \frac{\partial^{2} u}{\partial x_{i} \partial y_{i}}+\frac{l}{m} u^{(l / m-1)}(x, y) \frac{\partial u}{\partial x_{i}} \frac{\partial u}{\partial y_{i}}\right|^{m} d x_{i} d y_{i}\right)^{1 / m}
\end{gathered}
$$

which is unless $\int a_{i}{ }^{b_{i}} \int c_{i}{ }^{d_{i}}|u(x, y)|^{l+m} d x_{i} d y_{i}=0$ (for which the inequality (2.8) is obvious), is the same as 


$$
\begin{gathered}
\left(\int a_{i}{ }^{b_{i}} \int c_{i}{ }^{d_{i}}|u(x, y)|^{l+m} d x_{i} d y_{i}\right)^{1 / m} \leq \frac{m+l}{2 m}\left[\left(b_{i}-a_{i}\right)\left(d_{i}-c_{i}\right)\right] \\
\times\left(\int a_{i}^{b_{i}} \int c_{i}{ }^{d_{i}}\left|u^{l / m}(x, y) \frac{\partial^{2} u}{\partial x_{i} \partial y_{i}}+\frac{l}{m} u^{(l / m-1)}(x, y) \frac{\partial u}{\partial x_{i}} \frac{\partial u}{\partial y_{i}}\right|^{m} d x_{i} d y_{i}\right)^{1 / m} .
\end{gathered}
$$

Finally, raising $m$-th power both sides of the above inequality, integrating the resulting inequality from $a_{j}$ to $b_{j}$ and $c_{j}$ to $d_{j}$, respectively, then summing the $n$ inequalities $1 \leq i \leq n$, we find the desired inequality (2.8).

Remark 2.6. Let $u(x, y)$ reduce to $u(x)$ in (2.8) and with suitable modifications, then (2.8) becomes the following Agarwal and Sheng [15] inequality.

$$
\int E|u(x)|^{l+m} d x \leq \frac{1}{n}\left(\frac{m+l}{2 m}\right)^{m} \sum_{i=1}^{n}\left(b_{i}-a_{i}\right)^{m} \int E|u(x)|^{l}\left|\frac{\partial}{\partial x_{i}} u(x)\right|^{m} d x .
$$

Remark 2.7. The inequality (2.8) for $u(x, y)$ reduce to $u(x)$, with the right-hand sides multiplied by $m^{m}$ and $\left(b_{i}-a_{i}\right)^{m}$ replaced by $(\alpha \beta)^{m}$ has been obtained by Pachpatte [18].

\section{Acknowledgements}

C.-J. Zhao research was supported by National Natural Sciences Foundation of China (10971205). W.-S. Cheung research was partially supported by a HKU URG grant.

\section{Author details}

'Department of Mathematics, China Jiliang University, Hangzhou 310018, P. R. China ${ }^{2}$ Department of Mathematics, The University of Hong Kong, Pokfulam Road, Hong Kong ${ }^{3}$ Str. Hărmanului 6, 505600 Săcele-Négyfalu, Jud. Braşov,

Romania

\section{Authors' contributions}

C-JZ, W-SC and MB jointly contributed to the main results Theorems 2.1, 2.2, and 2.3. All authors read and approved the final manuscript.

\section{Competing interests}

The authors declare that they have no competing interests.

Received: 1 February 2012 Accepted: 18 May 2012 Published: 18 May 2012

\section{References}

1. Agarwal, RP, Pang, PYH: Opial Inequalities with Applications in Differential and Difference Equations. Kluwer Academic Publishers, Dordrecht (1995)

2. Agarwal, RP, Lakshmikantham, V: Uniqueness and Nonuniqueness Criteria for Ordinary Differential Equations. World Scientific, Singapore (1993)

3. Agarwal, RP, Thandapani, E: On some new integrodifferential inequalities Anal sti Univ. "Al I Cuza" din lasi. 28, 123-126 (1982)

4. Bainov, D, Simeonov, P: Integral Inequalities and Applications. Kluwer Academic Publishers, Dordrecht (1992)

5. Li, JD: Opial-type integral inequalities involving several higher order derivatives. J Math Anal Appl. 167, 98-100 (1992). doi:10.1016/0022-247X(92)90238-9

6. Mitrinovič, DS, Pečarić, JE, Fink, AM: Inequalities involving Functions and Their Integrals ang Derivatives. Kluwer Academic Publishers, Dordrecht (1991)

7. Cheung, WS: On Opial-type inequalities in two variables. Aequationes Math. 38, 236-244 (1989). doi:10.1007/ BF01840008

8. Cheung, WS: Some new Opial-type inequalities. Mathematika. 37, 136-142 (1990). doi:10.1112/S0025579300012869

9. Cheung, WS: Some generalized Opial-type inequalities. J Math Anal Appl. 162, 317-321 (1991). doi:10.1016/0022-247X (91)90152-P

10. Cheung, WS: Opial-type inequalities with $m$ functions in $n$ variables. Mathematika. 39, 319-326 (1992). doi:10.1112/ S0025579300015047

11. Crooke, PS: On two inequalities of the Sobolev type. Appl Anal. 3, 345-358 (1974). doi:10.1080/00036817408839076

12. Pachpatte, BG: Opial type inequality in several variables. Tamkang J Math. 22, 7-11 (1991)

13. Pachpatte, BG: On some new integral inequalities in two independent variables. J Math Anal Appl. 129, 375-382 (1988). doi:10.1016/0022-247X(88)90256-9

14. Wang, XJ: Sharp constant in a Sobolev inequality. Nonlinear Anal. 20, 261-268 (1993). doi:10.1016/0362-546X(93)90162-L

15. Agarwal, RP, Sheng, Q: Sharp integral inequalities in $n$ independent varibles. Nonlinear Anal Theory Methods Appl. 26(2):179-210 (1996). doi:10.1016/0362-546X(94)00273-K 
16. Pachpatte, BG: On Sobolev type integral inequalities. Proc R Soc Edinb. 103, 1-14 (1986). doi:10.1017/ S0308210500013986

17. Pachpatte, BG: On two inequalities of the Serrin type. J Math Anal Appl. 116, 193-199 (1986). doi:10.1016/0022-247X(86) 90051-X

18. Pachpatte, BG: On some variants of Sobolev's inequality. Soochow J Math. 17, 121-129 (1991)

doi:10.1186/1029-242X-2012-109

Cite this article as: Zhao et al:: Some sharp integral inequalities involving partial derivatives. Journal of Inequalities and Applications 2012 2012:109.

Submit your manuscript to a SpringerOpen ${ }^{\odot}$ journal and benefit from:

- Convenient online submission

- Rigorous peer review

- Immediate publication on acceptance

- Open access: articles freely available online

- High visibility within the field

- Retaining the copyright to your article

Submit your next manuscript at $\gg$ springeropen.com 